\title{
X-Ray and Energy Calculation Studies on the Packing-Mode of Double-Stranded Helices of Isotactic Poly(methyl methacrylate)
}

\author{
Hiroshi KUSANAGI
}

Unitika Research Laboratories Inc., Uji, Kyoto 611, Japan

(Received March 15, 1996)

\begin{abstract}
For isotactic poly(methyl methacrylate) (it-PMMA), structural reasons on the rather complicated crystal structure with the space group Fdd2 determined by X-Ray analysis were investigated. The X-Ray diffraction intensity distribution of the first layer line was found to reflect the locations of the (10/1) double-stranded helices along the $c$-axis. And the energy calculation indicated that the packing-mode of double-stranded helices accompanying the space group Fdd 2 is $\sim 2.0-$ $2.5 \mathrm{kcal} \mathrm{mol}^{-1}$ monomer ${ }^{-1}$ more stable than those with Pnn2, Pba2, and P2 $1 / \mathrm{a}$ as calculated by molecular mechanics energy calculations. For it-PMMA, the present X-ray study and crystal energy calculations support the rather complicated crystal structure.
\end{abstract}

KEY WORDS Molecular Mechanics Calculation / Poly(methyl methacrylate)/ Double-Stranded Helix / Packing-Mode / Stabilizing Energy /

After a long and somewhat roundabout quest the double-stranded helix was finally found in 1976 by the present author and coworkers for the molecular structure of isotactic poly(methyl methacrylate) (it-PMMA). ${ }^{1}$ The double-stranded helix structure, which was proposed then for the first time in synthetic polymers, had been accepted to be essentially correct from the results of many different kinds of studies, such as conformational energy calculations, ${ }^{2,3}$ Fourier-transform infrared spectroscopy, ${ }^{4}$ normal-mode calculations, ${ }^{5}$ and solidstate NMR spectroscopy. ${ }^{6}$ However, the crystal structure of it-PMMA had not been analyzed, therefore, its complete structure determination remained an open problem. ${ }^{7,8}$ Thereafter, the possibility of a non-helical molecular model was suggested, ${ }^{9}$ although this was largely to pose the question as to why the crystal structure cannot be soled by using the helix structure. Under such a situation, the author and coworkers spent a great effort to determine the crystal structure of it-PMMA, and so, by the aid of computer calculations, they had been able to setting up the accurate starting model for the molecular and crystal structure, and as the result, at last, recently succeeded to determine the crystal structure of it-PMMA and brought this open problem to an end. ${ }^{10}$

Molecular and crystal structure of it-PMMA are shown in Figures 1 and 2. The crystals are orthorhombic, with rather large cell dimension, i.e. $a=41.96 \AA, b=$ $24.34 \AA$, and $c$ (fiber axis) $=10.50 \AA$. There are eight $(10 / 1)$ double-stranded helices of the right-handed and left-handed senses in the unit cell, which take up a slightly complicated hexagonal-like closest packing-mode, and where among the first six neighbor helices the two same-handed senses are located at $z=c / 2$ and the four opposite-handed senses are located at $z=c / 4$ and $3 c / 4$.

The author is aiming to clarify the structural reasons for this complicated helical packing-mode of it-PMMA, and reports here results from X-Ray analysis and molecular mechanics calculations using the packing energy minimization method for polymers. ${ }^{11}$

\section{METHOD}

Crystal Structure Models, X-Ray Analysis, and Energy Calculations

It-PMMA as synthesized is an apparently racemic polymer, so that right-handed and left-handed helices may coexist in the crystal. Accordingly, during the process of the previous X-ray structure analysis, ${ }^{10}$ there were remained four kinds of possible space groups for the achiral crystal within right-handed and left-handed

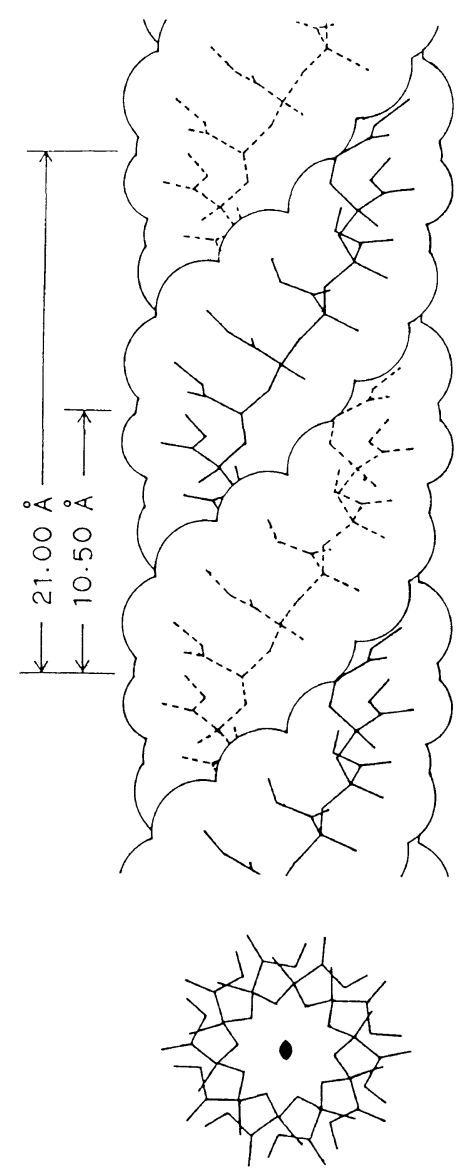

Figure 1. The (10/1) double-stranded helical molecular structure of it-PMMA. 


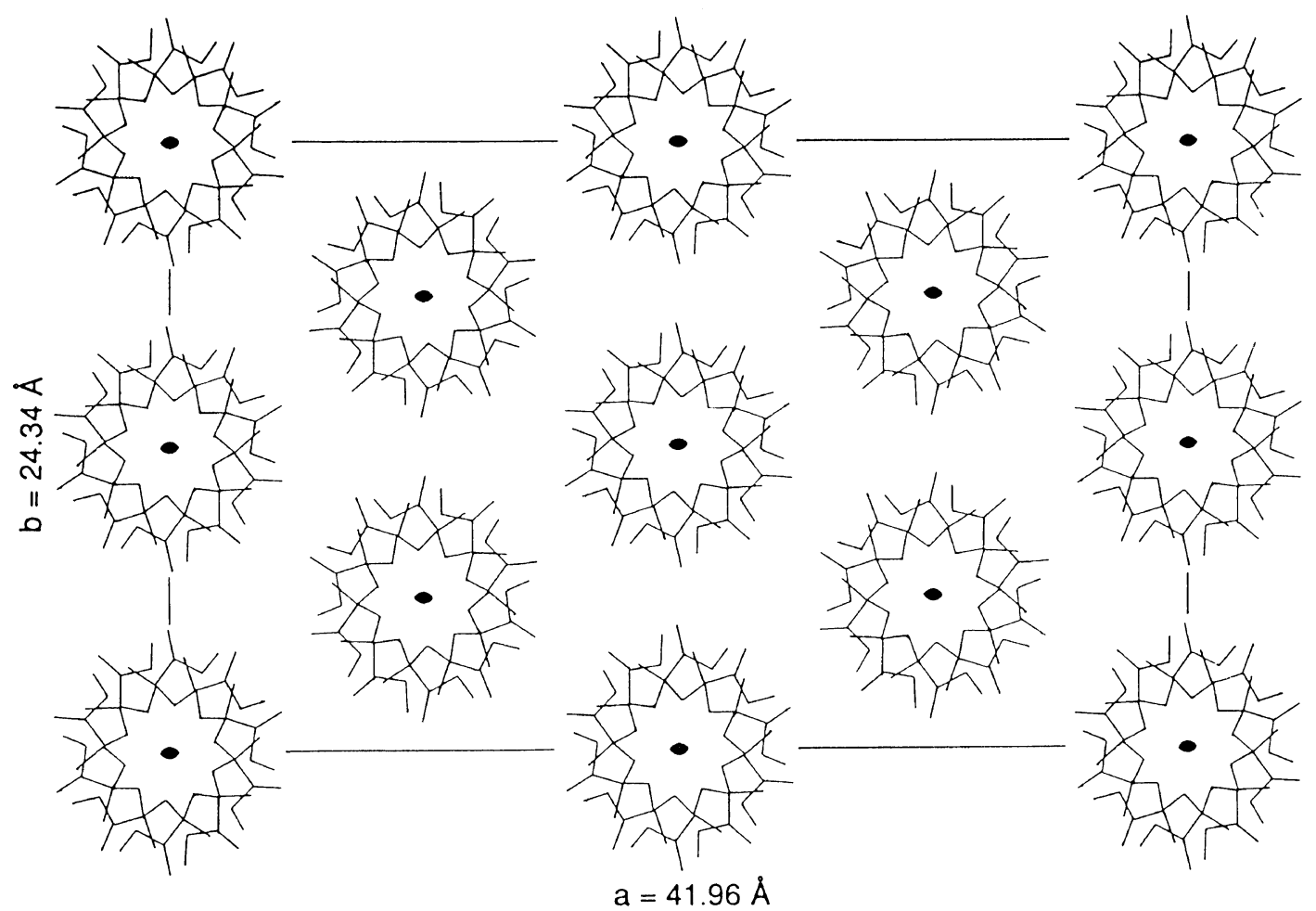

Figure 2. Crystal structure of it-PMMA.

Table I. Crystallographic parameters of the possible space groups of it-PMMA for the energy calculation

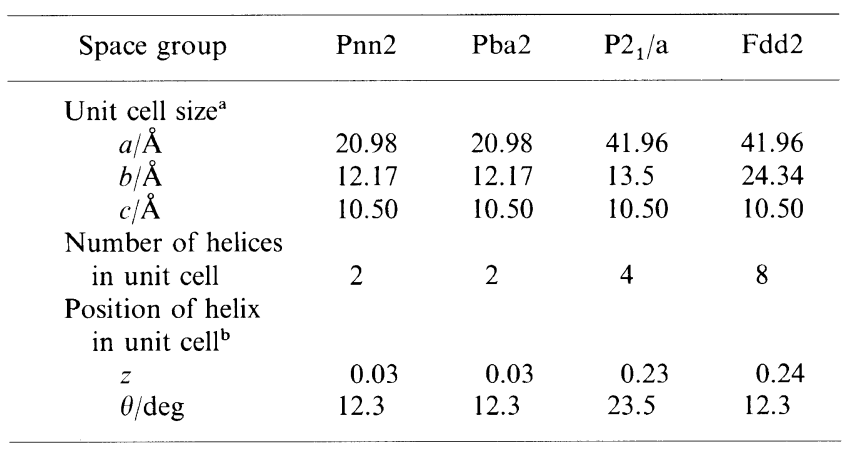

all lattice angles: $\alpha=\beta=\gamma=90^{\circ}$ except for $\alpha=111.33^{\circ}$ of $\mathrm{P} 2$ 1/a. ${ }^{\mathrm{b}}$ Fractional coordinates $(x, y, z)$ of helix axis; all $x=y=0$, the origin of $z$ is the height of the main chain carbon atom $\mathrm{Cl}$ denoted in Table II. $\theta$ is the setting angle between the $a$-axis and the direction from the $\mathrm{C} 1$ atom to helix axis.

$(10 / 1)$ double-stranded helices of it-PMMA. For these four kinds of the space groups, crystallographic data are listed in Table I. Among them, the large unit cell contains 4 times of double-stranded helices comparing with the small unit cell. These four crystal structure models were examined by using the X-Ray diffraction data and the crystal energy calculations. Molecular parameters of double-stranded helix used were the same as those by the previous X-Ray analysis and are listed in Table II. In X-ray analysis of the present study, the relation between the $\mathrm{X}$-ray diffraction intensity distribution on the first layer line and helical packing along the $c$-axis was examined. Energy calculations on the crystal structure were carried out with using the program of the packing energy minimization for polymers and the potential parameters. ${ }^{11}$ For the electrostatic interactions, point charges are adopted to ester groups and parameter set were used as the same and similar chemical
Table II. Molecular parameters of (10/1) helix of it-PMMA for the energy calculation

\begin{tabular}{|c|c|c|c|}
\hline \multicolumn{2}{|l|}{ Bond lengths/A } & \multirow{2}{*}{$\begin{array}{l}\mathrm{C} 3-\mathrm{C} 1-\mathrm{M} 2 \\
\mathrm{Cl}^{\prime}-\mathrm{C} 2-\mathrm{C} 1\end{array}$} & \multirow{2}{*}{$\begin{array}{l}105.5 \\
124.0\end{array}$} \\
\hline $\mathrm{C} 1-\mathrm{C} 2$ & 1.53 & & \\
\hline $\mathrm{C} 22^{\prime}-\mathrm{C} 1$ & 1.53 & $\mathrm{Cl}-\mathrm{C} 3-\mathrm{O} 1$ & 108.0 \\
\hline $\mathrm{C} 1-\mathrm{C} 3$ & 1.52 & $\mathrm{C} 1-\mathrm{C} 3-\mathrm{O} 2$ & 128.0 \\
\hline $\mathrm{C} 3-\mathrm{O} 1$ & 1.35 & $\mathrm{O} 1-\mathrm{C} 3-\mathrm{O} 2$ & 124.0 \\
\hline $\mathrm{C} 3-\mathrm{O} 2$ & 1.25 & $\mathrm{C} 3-\mathrm{Ol}-\mathrm{M} 1$ & 112.0 \\
\hline $\mathrm{O} 1-\mathrm{M} 1$ & 1.42 & Other angles & 109.5 \\
\hline $\mathrm{C} 1-\mathrm{M} 2$ & 1.54 & & \\
\hline $\mathrm{C}-\mathrm{H}$ & 1.10 & Torsion angles/deg & \\
\hline \multicolumn{2}{|c|}{ Bond angles/deg } & $\mathrm{C} 2^{\prime}-\mathrm{Cl}-\mathrm{C} 2-\mathrm{Cl}^{\prime \prime}$ & -161.8 \\
\hline $\mathrm{C} 2^{\prime}-\mathrm{C} 1^{\prime}-\mathrm{C} 2$ & 103.7 & $\mathrm{C}^{\prime}-\mathrm{C} 2^{\prime}-\mathrm{C} 1-\mathrm{C} 3$ & 75.4 \\
\hline $\mathrm{C} 22^{\prime} \mathrm{C} 1-\mathrm{C} 3$ & 106.0 & $\mathrm{Cl}^{\prime}-\mathrm{C} 2^{\prime}-\mathrm{C} 1-\mathrm{M} 2$ & -40.7 \\
\hline $\mathrm{C} 2{ }^{\prime}-\mathrm{C} 1-\mathrm{M} 2$ & 115.0 & $\mathrm{C} 22^{\prime}-\mathrm{C} 1-\mathrm{C} 3-\mathrm{Cl}$ & 63.0 \\
\hline $\mathrm{C} 2-\mathrm{C} 1-\mathrm{C} 3$ & 115.5 & $\mathrm{C} 1-\mathrm{C} 3-\mathrm{O} 1-\mathrm{M} 1$ & 190.0 \\
\hline $\mathrm{C} 2-\mathrm{C} 1-\mathrm{M} 2$ & 111.5 & $\mathrm{M} 2-\mathrm{C} 1-\mathrm{C} 3-\mathrm{O} 1$ & -174.7 \\
\hline
\end{tabular}

${ }^{\text {a }}$ The atoms are numbered as follows:

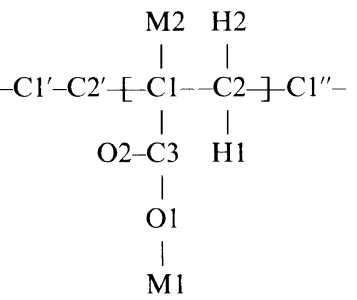

groups. ${ }^{12,13}$ The crystal energy $(E)$ is calculated for an unit of the crystallographic asymmetric unit (=five monomer units in an identity period of $c$-axis, $c=$ $10.5 \AA$ ), under the periodic boundary condition in three directions of the $a-, b$-, and $c$-axes. The crystal structure models with the molecular position determined by X-Ray analysis were used as the starting models, and the crystal energy was minimized with respect to the positional parameters of the double-stranded helix in the crystal 
lattice. Kubota RS3230 computer work station was used for the calculations.

\section{RESULTS AND DISCUSSION}

\section{$X$-Ray Analysis}

Figure 3 shows schematically the four space groups of packing-mode of the double-stranded helices. Two small unit cells with the space group Pnn2 and Pba2 contain two double-stranded helices of a right- and a left-handed helices, respectively. The difference between the Pnn 2 and $\mathrm{Pba} 2$ is the position of one double-stranded helix, which is located at the center of the unit cell, along the $c$-axis: $\operatorname{Pnn} 2 z=c / 2$ and $\mathrm{Pba} 2 z=0$. In the space group $\mathrm{P} 22_{1} / \mathrm{a}$ of medium unit cell within four doublestranded helices, there are four positions of helices along the $c$-axis and is a little complicated packing-mode comparing to the Pnn2 and Pba2. On the other hand, the true crystal structure of it-PMMA, which has the largest unit cell with Fdd2 space group, shows rather complicated packing-mode composed of eight doublestranded helices as mentioned as above. Among these four packing-modes, it was found in the final process of $\mathrm{X}$-ray analysis that the nature prefer the most complicated one. For them, Table III lists the discrepancy factor $R\left(=\Sigma\left|\sqrt{I_{0}}-\sqrt{I_{\mathrm{c}}}\right| / \Sigma \mid \sqrt{I_{0}}\right)$, where $I_{0}$ and $I_{\mathrm{c}}$ are, respectively, the observed and calculated X-ray diffraction intensities, which is the criterion of the reliability for the crystal structure in X-ray analysis. Reasonable value of $R$-factor is smaller than 0.20 for the crystal structure analyses of synthetic polymers. ${ }^{14}$ And

(a) Pnn2

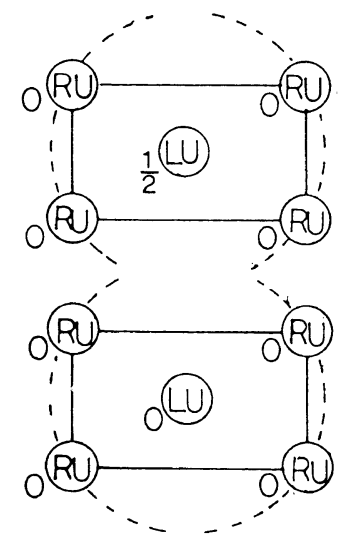

(b) Pba2

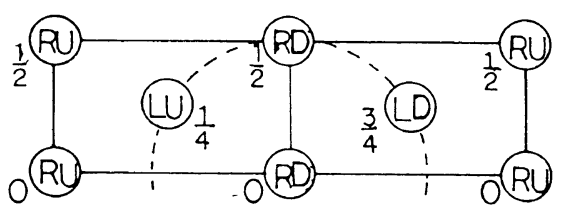

(d) Fdd2 (Cs)

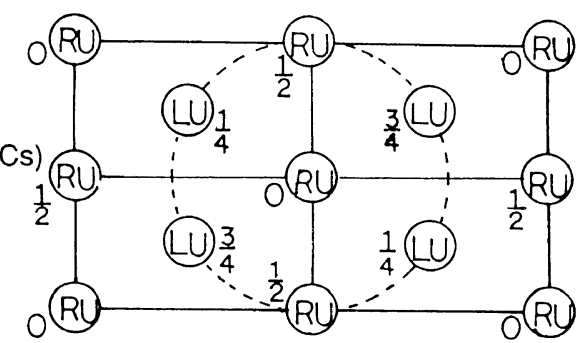

Figure 3. Schematic representations of the packing modes of doublestranded helices with respect to possible space groups. (a) Pnn2, (b) $\mathrm{Pba} 2$, (c) $\mathrm{P} 21 / \mathrm{a}$, and (d) Fdd2. the comparison between the observed and calculated intensities on the first layer line is shown in Figure 4. The simple packing of double-stranded helices accompanying the small unit cell are in bad agreement of the observed and calculated intensities. From these data, an intimate relationship between the degree of agreement in the first layer line and the positions along the $c$-axis occurs to the author's mind. In X-ray diffraction theory, the diffraction intensity distribution of the each layer line (in the reciprocal space) strongly reflect the packing of the double-stranded helices (in the real space). In this case, the diffraction intensity distribution of the first layer line may reflect the locations of the double-stranded helices along the $c$-axis. That is, the observed diffraction intensities reveal that the simple helical packing-mode of $z=0$ and $c / 2$ is not correct but the rather complicated packing-mode of $z=0, c / 4, c / 2$, and $3 c / 4$ is corrected. In the next section, this $\mathrm{X}$-ray result was examined by the energy calculations.

\section{Energy Calculations}

Table IV lists the results of energy calculations, and the dependency of crystal energy $E$ on the unit cell size and space group was considered. The large unit cell with space group Fdd 2 is the most stable one among the calculated crystal structure models, and the Fdd 2 crystal gives rather large stabilizing energy $\sim 10.1-12.4 \mathrm{kcal}$ $\mathrm{mol}^{-1}$ of crystallographic asymmetric unit ( 5 monomers) in comparing with the Pnn2, Pba2, and P2/a crystals. Therefore, the results of energy calculations support the crystal structure determined by X-ray analysis. An internal comparison of crystal energies reveals several

Table III. Dependency of $R$-factor on the unit cell size and space group

\begin{tabular}{lllll}
\hline \multicolumn{1}{c}{ Space group } & Pnn2 & Pba2 & P2 $1 / \mathrm{a}$ & Fdd2 \\
\hline $\begin{array}{llll}\text { Unit cell size } \\
\text { Number of helices }\end{array}$ & Small & Small & Medium & Large \\
$\begin{array}{l}\text { in unit cell } \\
R \text {-factor }\end{array}$ & 2 & 2 & 4 & 8 \\
& 0.41 & 0.36 & 0.35 & 0.21 \\
\hline
\end{tabular}
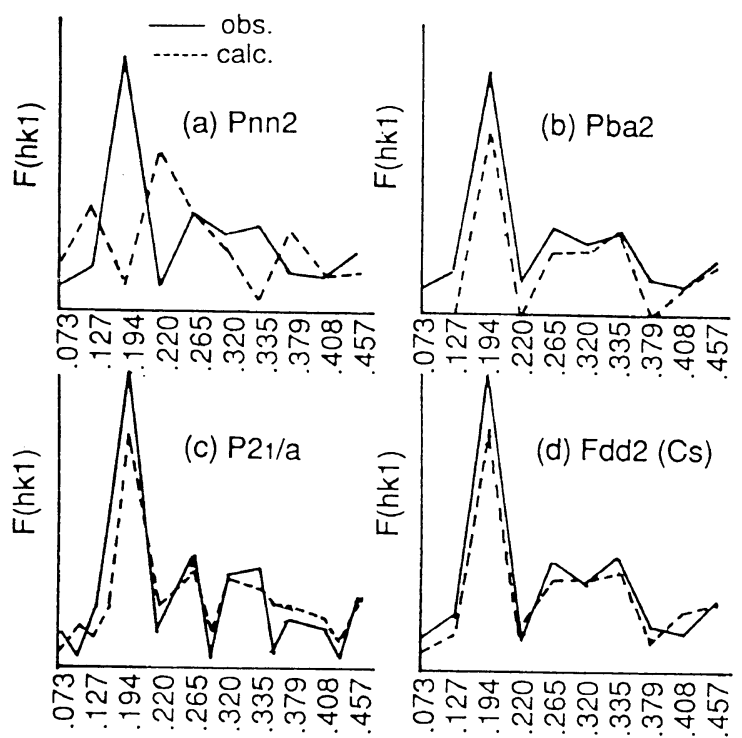

Figure 4. Dependency of the agreement between the observed and calculated X-ray reflection intensities in the first layer line on possible space groups. (a) Pnn2, (b) Pba2, (c) P2/a, and (d) Fdd2. 
Table IV. Dependency of crystal energy $E$ on the unit cell size and space group

\begin{tabular}{crrrr}
\hline Space group & Pnn2 & Pba2 & P2 $1 / \mathrm{a}$ & Fdd2 \\
\hline Unit cell size & Small & Small & Medium & Large \\
Crystal energy $^{a}$ & & & & \\
$E$ & -82.9 & -82.8 & -85.3 & -95.4 \\
$E_{\mathrm{vdw}}$ & -85.3 & -85.3 & -91.6 & -94.5 \\
$E_{\mathrm{el}}$ & 2.4 & 2.4 & 6.4 & -0.9
\end{tabular}

${ }^{\mathrm{a}} E$, total crystal enrgy; $E_{\mathrm{vdw}}$, van der Waals interaction energy; $E_{\mathrm{el}}$, the electrostatic interaction energy $\left(\mathrm{kcal} \mathrm{mol}^{-1}\right.$ of crystallographic asymmetric unit).

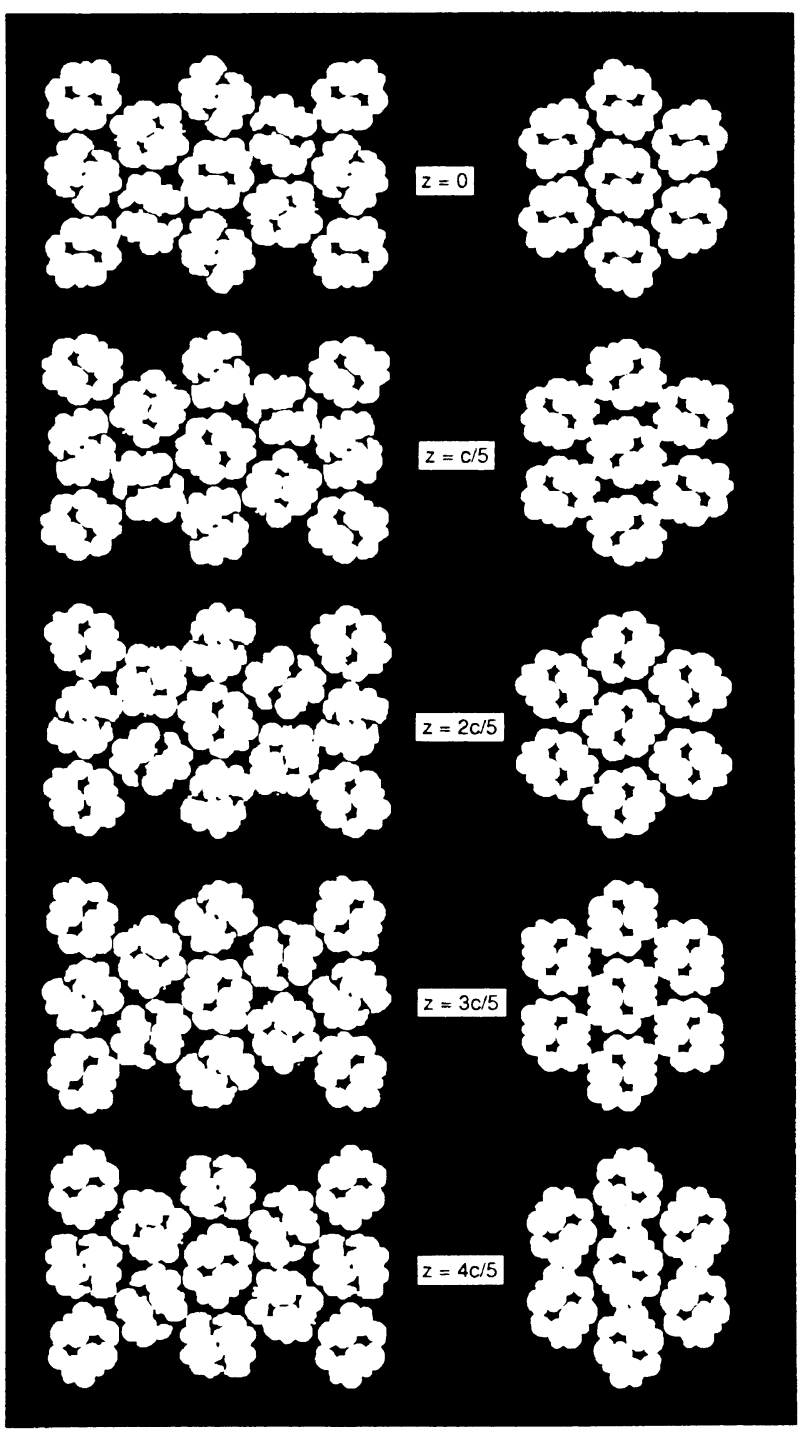

(a) Fdd2

(b) Pba2

Figure 5. The van der Waals contacts among the first neighbor double-stranded helices from the viewpoint of lattice sections from $z=0$ to $4 c / 5$. (a) Fdd2 (large unit cell) and (b) Pba2 (small unit cell).

insights. In the van der Waals interactions, the intermolecular attractive energy $\left(E_{\mathrm{vdw}}\right)$ of the small unit cell of simple packing-mode is the smallest, and the attractive energy $E_{\mathrm{vdw}}$ seems to increase with the extent of complicity in packing-mode. This may closely relate to the molecular symmetry. That is, the double-stranded helix of it-PMMA has two kinds of symmetries along the helix axis; (1) (10/1) helix symmetry and (2) two-fold rotation symmetry coinciding to the helix axis. The helical packing of such as two kinds of helix symmetries may be favor to take the complicated packing-mode of the space group Fdd2. This situation is understandable from the packing geometry, namely the visualization of the packing-mode of the strands. Figure 5 shows sliced sectional planes of the crystal lattice at $z=0$ to $4 c / 5$, with an interval of $\Delta z=c / 5$, presented in terms of the van der Waals molecular model. From these figures, it can be intuitively understood that the Fdd2 lattice has better packing-mode than the Pba2 lattice (of the samehanded sense) between the two adjacent double-stranded helices along the $b$-axis. This is because these two helices, which are located at the same height, come into close contact at $z=c / 5$ and $4 c / 5$, due to the two-fold rotation $\left(C_{2}\right)$ symmetry of the double-stranded helix.

As an example of the space group Fdd2, the crystal structure of poly(ethyleneimine) anhydrate has been known. ${ }^{15}$ And, it cannot be found up to now, except for it-PMMA and PEI, that such polymers possess the above two kinds of molecular symmetry. Therefore, it is concluded that it-PMMA and PEI have special and unique steric molecular structure. The helical structure of isotactic polypropylene has so the (3/1) helix symmetry but not the $C_{2}$ symmetry that its crystal structure may take the $\mathrm{P} 21 / \mathrm{a}$ space group with the secondary complicated helical packing-mode. ${ }^{16-18}$

Finally, the complicated helical packing-modes of it-PMMA and PEI seems to originate from (i) the fact that they are racemic polymers and take achiral crystal symmetry, and (ii) their double-stranded helices have both of the rotation and screw-rotation symmetries coinciding the helix axis.

In addition to the van der Waals interactions, we found, using the energy calculations, that the electrostatic interaction energy $\left(E_{\mathrm{el}}\right)$ appears to be important in stabilizing the Fdd2 crystal structure of it-PMMA over the other crystal structures with the Pnn2, Pba2, and $\mathrm{P} 2$ 1/a.

\section{REFERENCES}

1. H. Kusanagi, H. Tadokoro, and Y. Chatani, Macromolecules, 9, 531 (1976).

2. P. R. Sundraraajan, Macromolecules, 19, 415 (1986).

3. M. Vacatello and P. J. Flory, Macromolecules, 19, 405 (1986).

4. R. H. G. Brinkhuis and A. J. Schoutten, Macromolecules, 24, 1496 (1991).

5. J. Dybal and S. Krimm, Macromolecules, 23, 1301 (1990).

6. J. Spevacek, B. Schneider, and J. Straka, Macromolecules, 23, 3042 (1990).

7. V. M. Coiro, A. M. Liquori, P. De Santis, and L. Mazzarella, J. Polym. Sci., Polym. Lett. Ed., 16, 33 (1978).

8. R. Loveell and A. H. Windle, Macromolecules, 14, 211 (1981).

9. K. J. Miller, Macromolecules, 24, 6877 (1991).

10. H. Kusanagi, Y. Chatani, and H. Tadokoro, Polymer, 35, 2028 (1994).

11. H. Kusanagi, Kobunshi Ronbunshu, 53, 108 (1996).

12. D. R. Ferro, S. Bruckner, S. V. Meille, and M. Ragazzi, Macromolecules, 23, 1676 (1990).

13. S. J. Weiner, P. A. Kollman, D. A. Case, U. C. Singh, C. Chio, G. Alagona, S. Profeta, and P. Weiner, J. Am. Chem. Soc., 106, 765 (1984).

14. H. Tadokoro, "Structures of Crystalline Polymers," Wiley, New York, N.Y., 1979, p 159.

15. Y. Chatani, T. Kobatake, T. Tadokoro, and R. Tanaka, Macromolecules, 15, 170 (1982).

16. G. Natta and P. Corradini, Nuovo. Cimento Suppl., 15, 40 (1960).

17. Z. Mencik, J. Macromol. Sci., Phys., 6, 101 (1972).

18. M. Hikosaka and T. Seto, Polym. J., 5, 111 (1973). 